\title{
Sphingobacterium spiritivorum
}

National Cancer Institute

\section{Source}

National Cancer Institute. Sphingobacterium spiritivorum. NCI Thesaurus. Code C86960.

A species of aerobic, Gram negative, rod and cocci shaped bacteria assigned to the phylum Bacteroidetes. This species contains sphingolipids, can ferment glucose and mannitol, and is oxidase, DNase and urease positive. S. spiritivorum is most commonly pathogenic in immunocompromised individuals. 